\title{
Efficacy of a prevention program for eating disorders in schools: a cluster-randomized controlled trial
}

\author{
Antje Gumz ${ }^{1,3}$, Angelika Weigel ${ }^{1 *}$ (D, Anne Daubmann² ${ }^{2}$ Karl Wegscheider ${ }^{2}$, Georg Romer $^{4}$ and Bernd Löwe ${ }^{1}$
}

\begin{abstract}
Background: Previous prevention programs in the school context have not addressed both genders, have been time-consuming, or have had deficits in the evaluation method. The aim of the present study was to evaluate the impact of a universal prevention program for female and male adolescents on eating disorder pathology and related risk factors.

Methods: Between February 2012 and July 2014, 2515 students in 23 schools from 8th or 11th grade were assessed for eligibility in this longitudinal cluster-randomized controlled trial with a six months follow-up. Of those students, 2342 were cluster-randomized to the intervention condition which received a six school hours universal prevention program or to the no treatment control condition.

Results: The complete case population comprised 724 students in the intervention ( $54.3 \%$ female, $M=14.3$ years, $S D=1.61)$ and 728 in the control condition (57.0\% female, $M=14.7$ years, $S D=1.63)$. Random-effects analysis of covariance on the primary outcome showed no significant differences between the intervention and control groups in their eating disorder pathology change scores six months after the intervention. Regarding secondary outcomes, participants in the intervention group showed a greater increase in knowledge about eating disorders both after the intervention $(p<.001, E S=1.06)$ and six months later $(p=.01, E S=0.40)$. Greater reductions in anxiety severity were observed in the intervention group post-intervention $(p=.02$, ES $=0.22)$ which was not maintained at the six months follow-up. Results differed between participants from grade 8 and 11.
\end{abstract}

Conclusion: The present universal prevention program can be particularly recommended for adolescents from grade 11.

Trial Registration: ISRCTN 97989348

Keywords: Eating disorders, Prevention, Adolescents, Randomized controlled trial, Risk factors

\section{Background}

Adolescence is a vulnerable period regarding the development of an eating disorder [1]. The prevalence rates for this age are $2 \%$ for anorexia nervosa, $1 \%$ for bulimia nervosa and $2 \%$ for binge eating disorder [1]. Substantially more adolescents are affected by eating disorder symptomatology, i.e., dieting, weight loss behaviors [2, 3] or restrained eating and weight concerns [4]. Once full-

\footnotetext{
* Correspondence: a.weigel@uke.de

${ }^{1}$ Department of Psychosomatic Medicine and Psychotherapy, University Medical Center Hamburg-Eppendorf \& Schön Clinic Hamburg Eilbek, Germany, Martinistraße 52, 20246 Hamburg, Germany

Full list of author information is available at the end of the article
}

blown eating disorders emerge, the initiation of a recommended treatment is often delated [5] and particularly anorexia nervosa exhibits a high risk of chronicity [6]. Against this background, an implementation of eating disorder prevention programs during adolescence is particularly important [7].

Different considerations plead for an implementation of eating disorder prevention programs in the classroom setting during adolescence. From a theoretical point of view, female and male adolescents make up an important part of the social environment for each other [8]. Including complete classes as the most relevant peer group provides the opportunity to increase awareness of 
the adverse effect of peer pressure on eating disorder development [9]. Peers influence the extent of pressure to suit Western beauty ideals, individual appearance related attitudes and body dissatisfaction which are all risk factors for eating disorders [10, 11]. Additionally, an overlap of further eating disorder risk factors such as a low self-esteem and negative affects like depression and anxiety has to be expected in female and male adolescents $[10,11]$. From a practical point of view, it is easier for schools to deliver prevention programs to whole classes as most schools are co-educational.

Prevention programs delivered to whole classes follow a universal prevention approach as female and male adolescents participate without prior screening and regardless of risk status. In the field of eating disorders, the efficacy of universal prevention programs in terms of effect sizes is typically lower than in selective approaches [12] which has been attributed to different baseline scores in unselected samples [13]. Bailey and colleagues summarized the results of 46 trials of universal prevention programs for eating disorders as well as six metaanalytic reviews [14]. Satisfactory effect sizes were observed for outcomes related to eating disorder knowledge while risk factor related outcomes were barely improved. The authors concluded that research opportunities for universal eating disorder prevention could be found in following a cognitive dissonance rather than a psychoeducational approach and by offering interactive interventions in a multi-session format. Another narrative review on effective eating disorder prevention programs underlined the need for universal prevention programs that address both genders [15].

A most recent randomized controlled trial compared three eight lesson universal prevention programs that addressed females and males with an average age of 13 years and aimed at reducing both eating disorder and obesity risk factors [16]. In one condition most encouraging findings were observed with regard to risk factor reduction in female and male participants (i.e. Media Smart). However, there was evidence of iatrogenic effects of the other two conditions (i.e. Life Smart, HELPP). In these conditions, the authors observed a significant increase of eating concerns and perceived pressure to be thin at the six months follow-up in female participants relative to the control condition (i.e. HELPP) and worsened scores on four eating disorder risk factor variables in female participants as well as worsened scores on three eating disorder risk factor variables in male participants (i.e. Life Smart).

Despite the promising evidence of some universal prevention programs most of these programs do not manage to be transferred from research into classroom practice on the long run [17]. Current universal prevention programs for eating disorders suffer from several shortcomings. A duration of six to nine weekly lessons is too long to suit the demands of school curricula for each school year. In other cases, scientific evaluations of the programs do not satisfy methodological standards regarding sample size $[18,19]$, randomization [20] and follow-up assessments [21, 22]. Furthermore, in most instances to date, and with some notable expectations (e.g. [23]) stakeholders including professional organizations that are in regular contact with schools are not yet sufficiently involved in the program development, implementation and dissemination to improve sustainability [24]. Thus, methodologically rigorous efficacy trials of short universal prevention programs which address female and male adolescents and fit the school curricular are urgently needed [13].

Against this background, this cluster-randomized controlled trial was designed to evaluate the impact of a brief universal prevention program on eating disorder pathology and related risk factors for female and male adolescents. We hypothesized that students who participated in the program would exhibit a significantly greater decrease in eating disorder pathology six months after participation than students who did not participate (primary outcome). We further expected this group difference to emerge directly after participation. Additionally, we hypothesized that students who participated in the intervention would more extensively increase their knowledge of eating disorders, show greater improvements in their self-concept, exhibit less internalization of Western beauty ideals, and report reduced levels of depression and anxiety compared with the outcomes for the control group both immediately after and six months after the intervention. Finally, we performed mediation analyses and subgroup analyses to describe the intervention's effect in subgroups based on age and sex.

\section{Methods \\ Study design and participants}

The efficacy of the present eating disorder pathology reduction program was evaluated using a cluster-randomized controlled trial design.

All 144 secondary schools in the city of Hamburg, Germany, were invited to participate. Of those, ten schools actively refused to participate, and 111 did not respond to contact attempts. Thus, participants were recruited from $n=23$ secondary schools between February 2012 and July 2014. Eligible participants were students in the 8th or 11th grades (age 14 and 17 years) who provided written informed consent. Additional written informed consent from a parent or legal guardian was required for participants under 16 years of age. Insufficient knowledge of German language was the only exclusion criterion. 
The study was approved by the Ethics Committee of the Psychotherapist Chamber of Hamburg, Germany (July 26, 2011), as well as the Hamburg supervisory school authority. The study protocol has been published (see [25] or Additional file 1 available online), and the study was registered with the ISRCTN registry (ISRCTN 97989348).

\section{Randomization}

Participating schools were randomized into the study conditions (intervention vs. control) according to the order of application. Randomization was conducted by an external institution (Department of Medical Biometry and Epidemiology, University Medical Center HamburgEppendorf). Random allocation was performed in a 1:1 ratio stratified by school type (district vs. secondary school) and the socio-economic status of the school environment without blocking. During recruitment, several individual schools were combined with each other due to changes in the Hamburgian school system. As a consequence, the standardized socio-economic indicators of the individual schools were no longer applicable. Thus, random allocation had to be revised, and the result was an imbalance of schools in the study conditions. Once assigned, each school stayed in the same study condition for the entire study duration.

Before providing written informed consent, students and their parents or legal guardians obtained a letter of consent with a detailed description of the allocated study condition. Thus, participants could not be blinded to their study condition.

Participants in the two conditions received neither incentives nor feedback about individual test results. However, participants received a flyer with counselling and assistance services and were given the opportunity to contact a psychotherapist from the research team in case of any mental health issues.

\section{Outcome measures}

There were three times of assessment: 1) one month prior to the intervention (baseline), 2) directly after the intervention (post), and 3) six months after the intervention (follow-up). Parallel time points were used for assessments in the control group. At all times of assessment, outcomes were assessed using a questionnaire set with self-report items. At baseline, demographic characteristics and body mass index (BMI) were assessed.

The primary outcome was changes in eating disorder pathology from baseline to the six months follow-up. Following the suggestions by Wilksch [17] the global mean score of the children's version of the Eating Disorder Examination Questionnaire (Ch-EDE-Q; [26]) was used to assess the primary outcome. The Ch-EDE-Q total score is calculated from the mean score of 22 items which refer to eating disorder symptomatology occurrence in the past 28 days (range $0-6$, with greater scores indicating more frequent experience of symptoms). The criterion validity of this questionnaire has been demonstrated in multiple studies and languages and there is empirical support from community-based samples for the temporal stability of the EDE-Q subscale scores over five to fourteen months [27]. The inner consistency of the Ch-EDE-Q global mean score in the present sample was Cronbach's $\alpha=.955$.

The secondary outcome scales included a questionnaire to measure knowledge about eating disorders (20 questions covering different aspects of eating disorder risk factors and symptoms, with response options including "right", "wrong" and "don't know"; range 0-20). The Sociocultural Attitudes Towards Appearance Questionnaire (SATAQ-G; [28]) was used to measure three aspects of the internalization of Western beauty ideals. The psychometric properties of this gender-adapted questionnaire have been established in adolescent samples of studies in different countries [29, 30]. The inner consistencies of the SATAQ-G subscales ranged between Cronbach's $\alpha=.822$ (awareness subscale) to .900 (internalization subscale). The Multidimensional Self-Concept Scale (MSCS; [31, 32]) was used to measure selfconcept. In the present sample Cronbach's $\alpha$ was 921. The well-validated Patient Health Questionnaire-9 (PHQ-9; [33, 34]) and Generalized Anxiety Disorder Scale (GAD-7; [35, 36]) were used to measure the severity of depression and anxiety symptoms. Inner consistencies of these instruments were Cronbach's $\alpha=.819$ (PHQ-9) and $\alpha=.860$ (GAD-7) in the present sample.

\section{Intervention}

The inclusion of female and male adolescents at all levels of eating disorder risk without prior screening, the effort to include as many schools as possible, the focus on the reduction of risk for eating disorders and the promotion of protective factors as well as the inclusion of input from community stakeholders (Center of the Prevention of Addiction, CPA) were features which formed the universal approach of the present program [37]. The program comprised three lessons which were delivered over two consecutive weeks (270 min dose in total). Females and males engaged together in the present program. Figure 1 provides an overview of the content and course of the intervention.

During the first 90-min-lesson students defined and critically discussed Western beauty ideals for female and male adolescents. This intervention was followed by an individual reflection of own investments to follow beauty ideals and a media literacy intervention focusing on the influence of social media and advertisements on the individual pressure to suit Western beauty ideals and how to cope with this pressure. The first lesson ended with an 


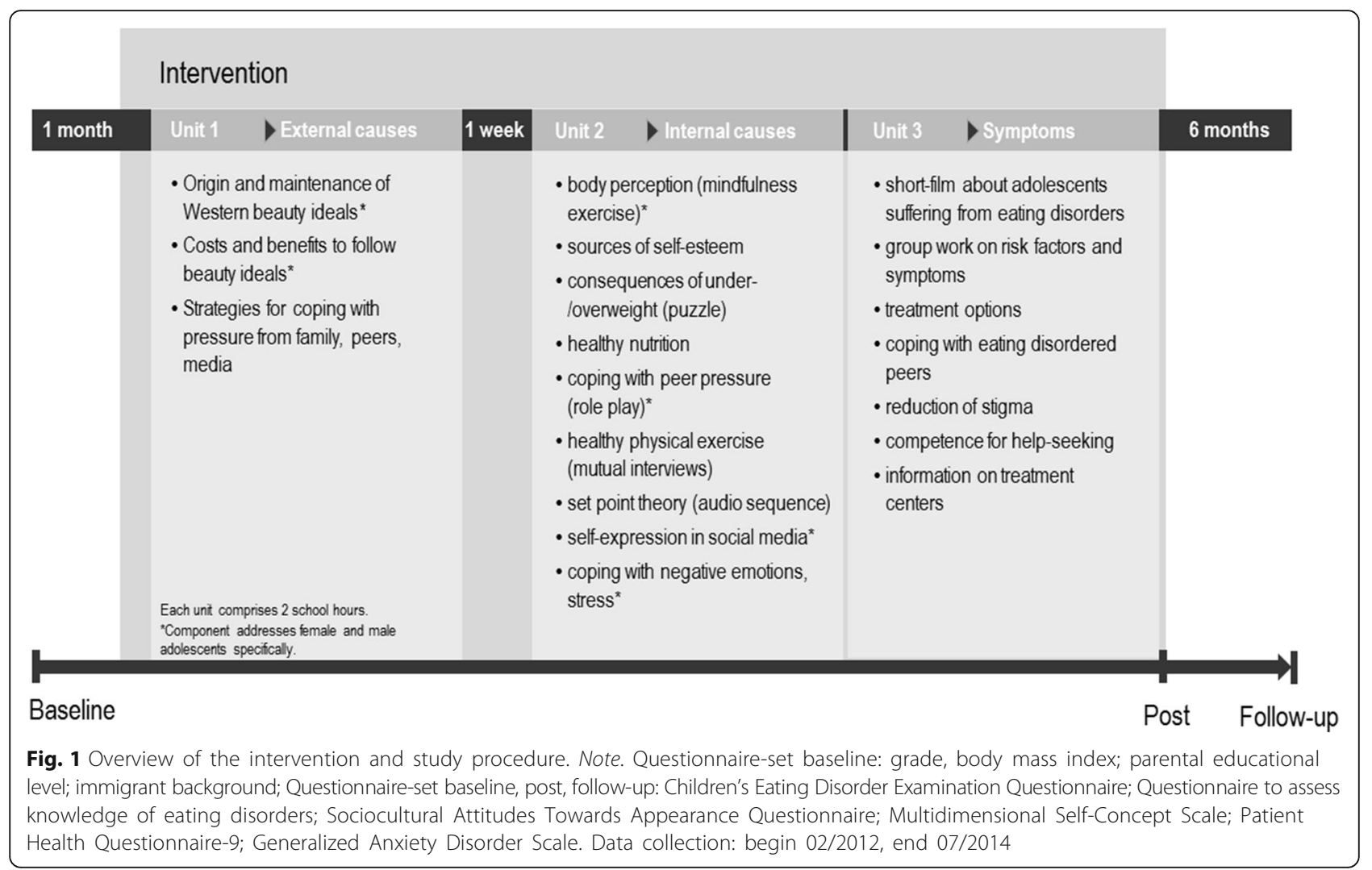

interactive intervention to help adolescents to cope with pressure from peers and family. One week later, female and male students past the second 90-min-lesson which comprised an interactive nine station parcour which addressed risk factors for eating disorders, protective factors and healthy nutrition (see Fig. 1). At each station, participants were provided with gender-adapted materials, e.g. different instructions for female and male participants. Adolescents passed each station in couples to allow for focused and open discussions. The third lesson directly followed the second lesson and was used to increase students' awareness towards local and online help-seeking in case of mental health issues as well as to develop coping strategies for the whole class if a class mate suffers from an eating disorder. A detailed description of the prevention materials and the dissonance-based approach of the prevention program is provided in the study protocol [25]. The prevention materials were piloted in one school in January 2012. As a result, instructions for some interventions were revised. A prevention manual was then created to standardize the oral and written information during implementation. The intervention was conducted between February 2012 and January 2014.

\section{Statistical methods}

The sample size was calculated for the prespecified contrast in the primary outcome, i.e., the adjusted mean difference in the ChEDE-Q change scores between the intervention and control groups from baseline to followup [38]. Change scores were applied to handle baseline differences between intervention and control group.

For a two-sided test with a type I error rate of $5 \%$, power of $80 \%$ and an effect size $(E S)$ of Cohen's $d=0.30$ [12], two samples with 176 participants each $(N=352)$ are needed to reach statistical significance. With an expected intracluster correlation of .05 and an average cluster size of 65 participants, the design effect is 4.6, which increased the required sample size to 739 participants per group $(N=1478)$. With an additional $25 \%$ loss to follow-up, a total of 1848 participants needed to be recruited.

We assessed baseline descriptive statistics for all randomly assigned participants by treatment allocation. The primary outcome hypothesis was tested using a randomeffects analysis of covariance (ANCOVA; [39]) with school as a random factor; with group (intervention vs. control), sex (male vs. female) and grade (8th vs. 11th) as fixed factors; and with baseline eating disorder risk as a continuous covariate using the complete case population at follow-up (although parental educational level appeared in the study protocol, it was not included as a fixed factor because $31 \%$ of the participants did not know their parents' educational level). The intracluster correlation coefficient, a measure of the heterogeneity of 
the average responses between schools, was calculated and tested based on the random terms of the model.

In a secondary analysis, we conducted an analogous random-effects repeated-measures ANCOVA at the post-intervention and follow-up points for each prespecified secondary outcome while allowing for heteroscedasticity and autocorrelation. In each case, the time of assessment (post, follow-up) was included as a repeated measure, and the significance of the time"group interaction was examined. These secondary analyses were adjusted for the influence of baseline levels from the respective secondary outcome. Preliminary analyses revealed significant differences between students who did not complete follow-up assessments and students who were "completers". In addition to the control variables documented in the study protocol, BMI and baseline eating disorder risk were included as continuous control variables in all analyses.

As post hoc subgroup analyses of the primary endpoint, a dummy variable including time (post, follow-up) and grade (8th vs. 11th) was coded, and the group*(time/grade) and sex"(time/grade) interactions were analyzed.

An additional secondary analysis aimed to improve the understanding of the interdependencies between the primary and secondary outcomes. Therefore, when a significant effect in the secondary outcomes was observed, the SPSS markro PROCESS [40] was used to analyze a potential mediating effect on the primary endpoint. These secondary analyses were first conducted in the complete case sample and then repeated in the subsamples of participants from grade 8 and 11, respectively. All other analyses were first performed on completers with subsequent sensitivity analyses in the intention-to-treat (ITT) population (participants with a baseline ChEDE-Q score). The multiple-imputation algorithm provided by SPSS was used to address missing values [41].

Two-tailed $p$-values $<.05$ were considered significant. Adjusted means with $95 \%$ confidence intervals (CIs) are represented in figures and tables. The data were analyzed using IBM SPSS Statistics version 21.0. and SAS Version 9.4 (SAS Institute Inc., Cary, NC, USA) was used for randomization.

\section{Results}

A total of 23 schools agreed to participate in the study. Of those, 15 schools ( $n=1187$ students) were randomly assigned to the intervention condition and eight schools ( $n=1155$ students) to the control condition $(N=2342$; Fig. 2). The participation rates for the intervention group were $88 \%$ at post-intervention and $86 \%$ at follow-up as

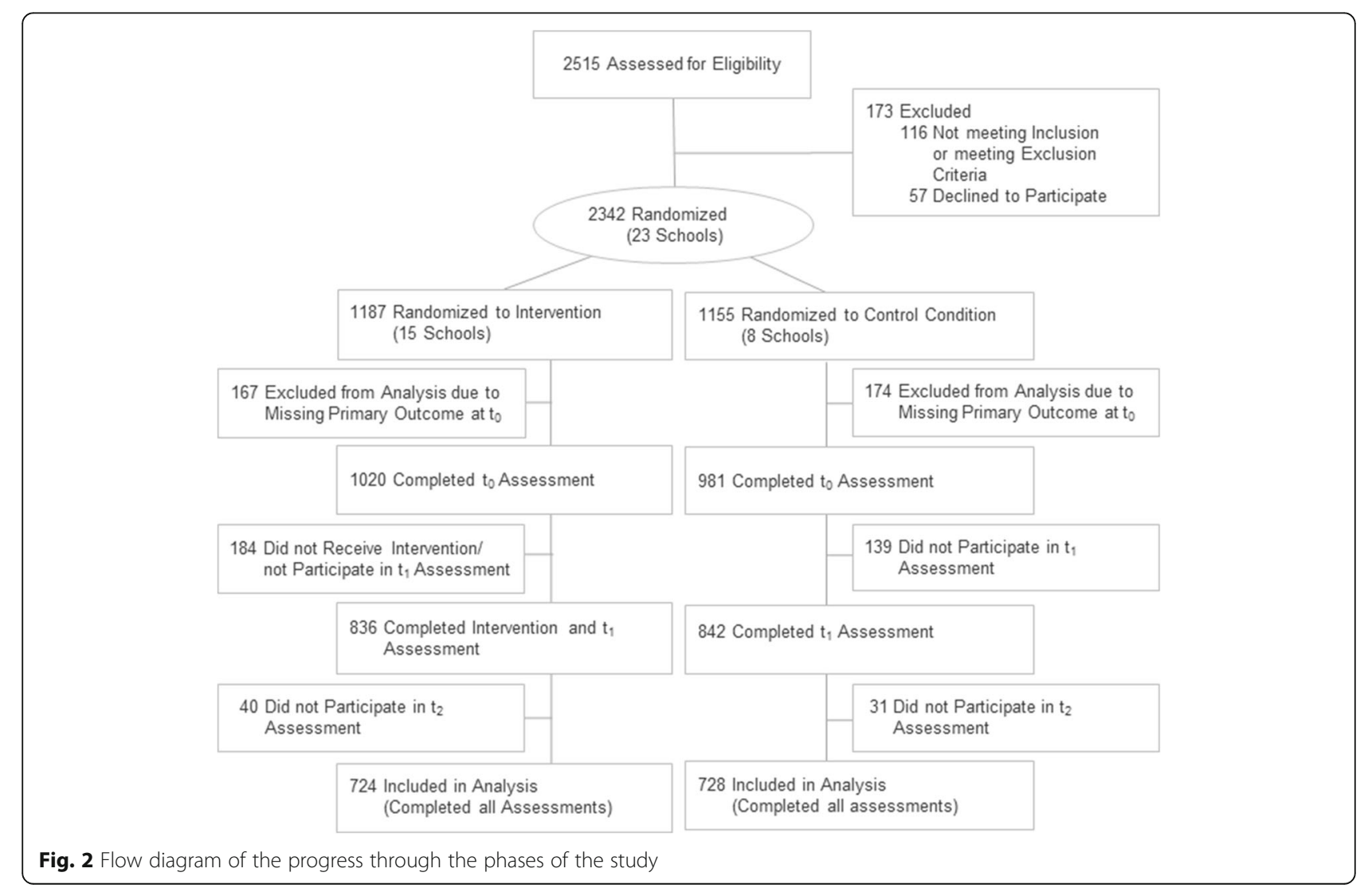


well as $85 \%$ and $89 \%$ for the control group. In grade 11 , frequencies of male and female students who did not complete follow-up assessments ("non-completers") did not significantly differ ( $30.3 \%$ vs.26.7\%; $X^{2}=1.245$, $p=.26)$. However, in grade 8 , significantly more male students were "non-completers" compared to female students $\left(30.4 \%\right.$ vs. $\left.23.8 \% ; X^{2}=6.829, p=.01\right)$.

Intervention and control group participants were well balanced with respect to all sociodemographic variables (Table 1).

In contrast to our primary hypothesis, we did not observe a significantly greater decrease in eating disorder pathology in the intervention group compared to the control group at follow-up (Table 2). However, in line with our secondary hypotheses, the intervention group displayed a significantly greater increase in eating disorder related knowledge than the control group after the intervention and at follow-up. Moreover, compared with the control group, the intervention group showed significantly greater improvements in anxiety symptoms and a greater increase in experienced pressure due to the internalization of Western beauty ideals directly after the intervention but not at follow-up. The intervention group did not exhibit superior change scores regarding self-concept and depression symptoms at either time of assessment (Table 2).

Taking into account the secondary outcomes and times of assessment in which a significant effect was observed, mediation models in the complete case sample revealed that changes in eating disorder knowledge at follow-up but not at post-intervention as well as changes in anxiety and pressure to suit Western beauty ideals at post-intervention partially mediated the relation between group allocation and changes in eating disorder pathology (Fig. 3). However, different indirect effects were observed in grades 8 and 11. In grade 8 , changes in pressure to suit Western beauty ideals at post-intervention $(\beta=0.033, B C I$ 95\% [0.013; 0.061], $z=3.070, p=.002)$ and changes in eating disorder knowledge $(\beta=0.024$, $B C I$ 95\% [0.007; 0.049], $z=2.240, p=.025)$ mediated the relation between group allocation and changes in eating disorder pathology. In grade 11, changes in anxiety at post-intervention $(\beta=-0.061, B C I$ 95\% [-0.129; -0.026], $z=-3.826, p=<.001)$ mediated the relation between group allocation and changes in eating disorder pathology.

Accordingly, we observed a significant interaction between participants' group allocation, time of assessment and grade in the post hoc analyses (complete case sample $p=.01$, ITT sample $p=.049$ ). As Fig. 4 illustrates, 11th grade female and male participants in the intervention group exhibited a lower eating disorder pathology than the participants in the control group at follow-up which was partially in line with our hypotheses, while 8th grade participants showed the opposite trends (interaction $p=.012$ ).

A second significant interaction between participants' sex, time of assessment and grade (complete case sample $p=<.001$, ITT sample $p=<.001$ ) indicated that in both groups and at both time points, 8th grade females

Table 1 Baseline characteristics of the complete case sample separated by group (intervention and control; $N=1452$ ) and comparison of completers vs. non-completers $(N=2001)$

\begin{tabular}{|c|c|c|c|c|c|c|}
\hline Characteristic & $\begin{array}{l}\text { Intervention } \\
n=724\end{array}$ & $\begin{array}{l}\text { Control } \\
n=728\end{array}$ & $p$ & $\begin{array}{l}\text { Completer } \\
n=1452\end{array}$ & $\begin{array}{l}\text { Non-Completer } \\
n=549\end{array}$ & $p$ \\
\hline Sex & & & .38 & & & .005 \\
\hline Female - n (\%) & $393(54.3)$ & $415(57.0)$ & & $808(55.6)$ & $268(48.8)$ & \\
\hline $\mathrm{Age}^{\mathrm{a}}-\mathrm{M}(\mathrm{SE})$ & $14.4(0.30)$ & $14.8(0.35)$ & .45 & $14.6(0.22)$ & $14.9(0.23)$ & .002 \\
\hline$B M I^{a}-M(S E)$ & $19.8(0.24)$ & $20.0(0.28)$ & .61 & $19.9(0.17)$ & $20.2(0.19)$ & .003 \\
\hline Grade & & & .62 & & & .55 \\
\hline 8th grade $-\mathrm{n}(\%)$ & $491(67.8)$ & $414(56.9)$ & & $905(62.3)$ & $333(60.7)$ & \\
\hline Migration background ${ }^{a}$ & & & .34 & & & .25 \\
\hline No migration background - n (\%) & $487(68.1)$ & $513(72.1)$ & & 1000 (69.6) & $347(64.6)$ & \\
\hline One parent - n (\%) & $118(16.5)$ & $86(11.9)$ & & $204(14.2)$ & $86(16.0)$ & \\
\hline One parent and participant or both parents - $\mathrm{n}(\%)$ & $92(12.9)$ & $88(12.2)$ & & $180(12.5)$ & $82(15.3)$ & \\
\hline Both parents and participant - $\mathrm{n}(\%)$ & $18(2.5)$ & $34(4.7)$ & & $52(3.6)$ & $22(4.1)$ & \\
\hline Parents educational level ${ }^{a}$ & & & .87 & & & .55 \\
\hline Low both parents - $\mathrm{n}(\%)$ & $78(15.8)$ & $92(18.2)$ & & $170(17.0)$ & $88(23.6)$ & \\
\hline High one parent - n (\%) & $94(19.1)$ & $124(24.5)$ & & $218(21.8)$ & $79(21.2)$ & \\
\hline High both parents - n (\%) & $321(65.1)$ & $290(57.3)$ & & $611(61.2)$ & $206(55.2)$ & \\
\hline
\end{tabular}

Note: ${ }^{2}$ Variable contains missing values. Percentages may be calculated using different denominators due to missing values. Non-completers are students who did not complete the follow-up assessments; completers are students who completed all follow-up assessments 


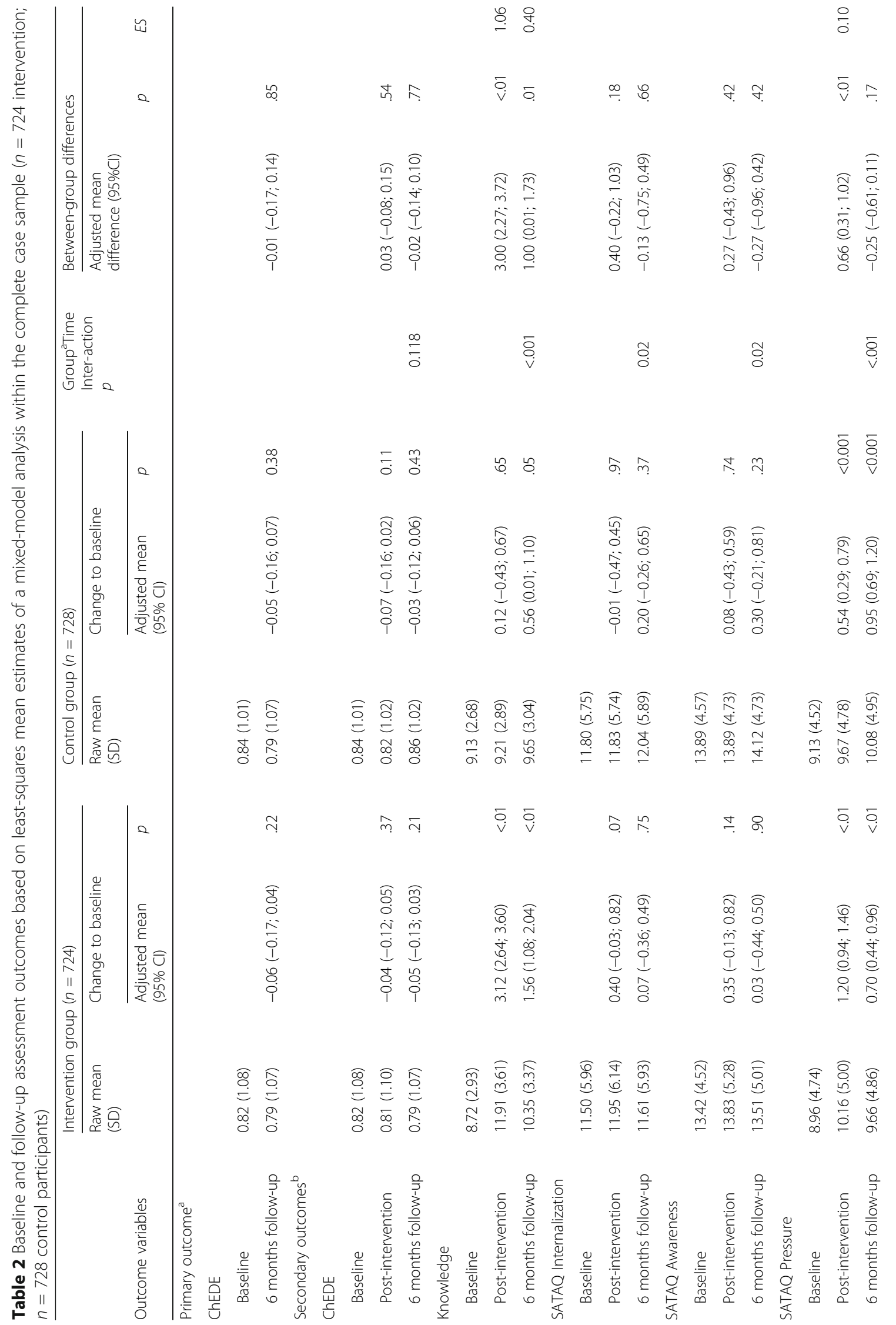


Gumz et al. BMC Psychiatry (2017) 17:293

Page 8 of 13

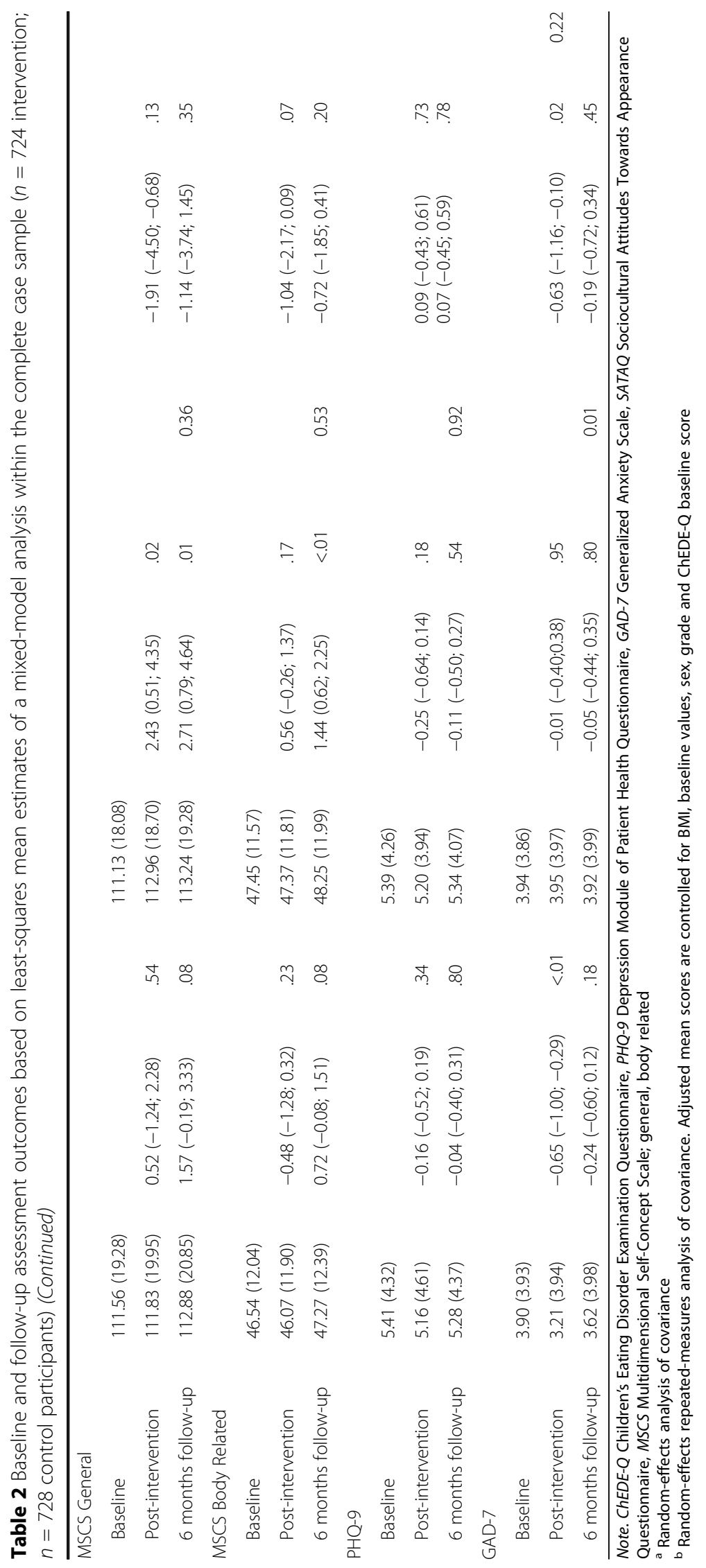




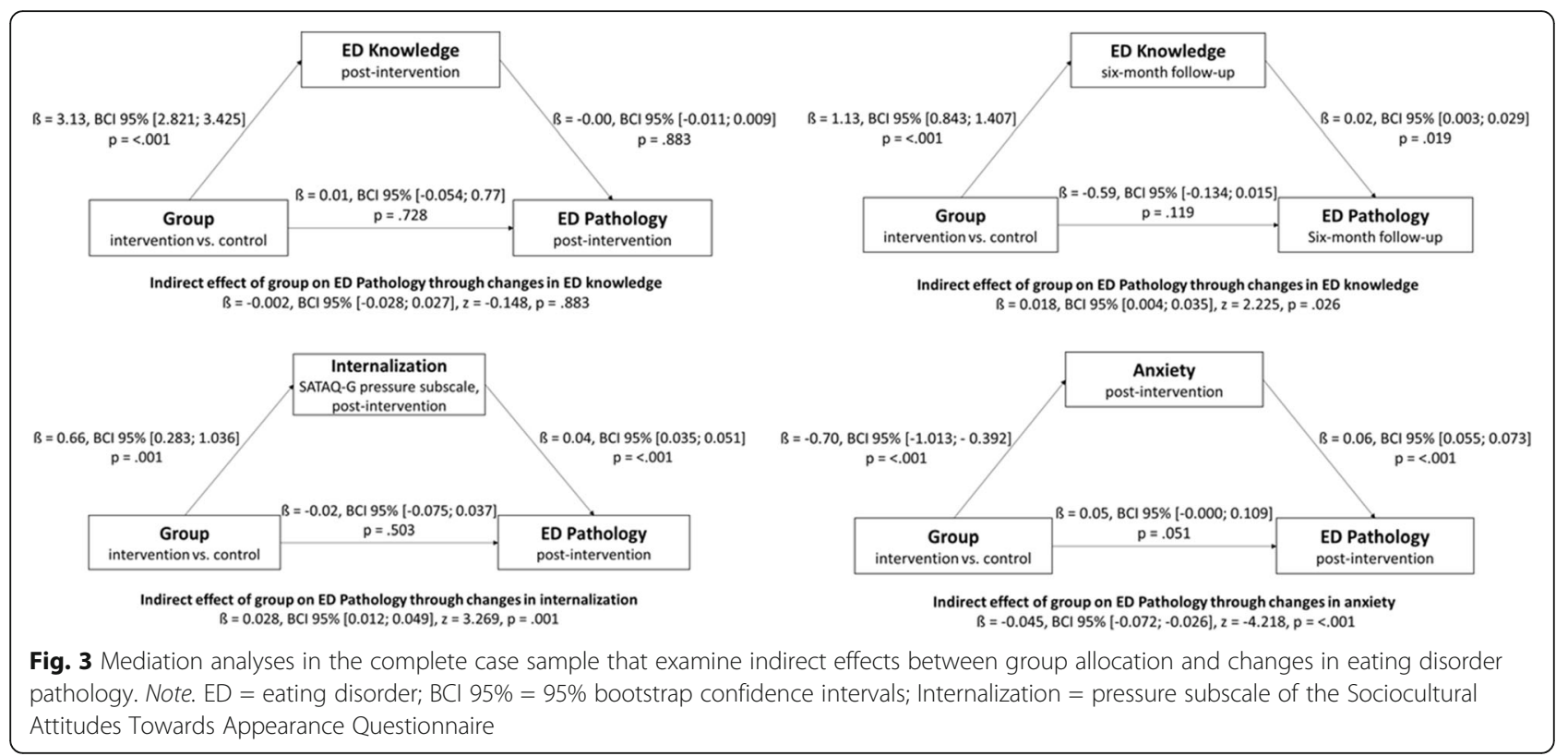

exhibited an increased eating disorder pathology, whereas in 8th grade males, the risk decreased directly after the intervention and subsequently remained stable (interaction $p=<.001$ ).

ITT analyses confirmed the complete case results (see Additional file 2: Table S1 available online).

\section{Discussion}

This cluster-randomized controlled trial was designed to evaluate the impact of a three lesson prevention program on eating disorder pathology for female and male adolescents. Regarding the primary endpoint, we did not find the expected difference in the change in eating disorder pathology between the intervention and control groups at six months follow-up. Regarding the secondary endpoints, adolescents who participated in the intervention as expected exhibited a persistent and larger increase in knowledge at both times of assessment compared to the control group which is in line with a previous meta-analytic review that observed higher effect sizes for outcomes related to eating disorder knowledge [12, 42]. Additionally, adolescents who participated in the intervention displayed a greater decrease in anxiety post-intervention compared with the control group. In contrast to our expectations, levels of depression and self-esteem were not positively influenced by the intervention. Moreover, the intervention group showed a greater increase in experienced pressure due to the internalization of Western beauty ideals directly after the intervention. This increase might be attributed to the increased awareness towards the presence of and pressure from Western beauty ideals and interpreted as a consequence of cognitive dissonance. Taken together, the present universal prevention program for eating disorders resulted in some positive effects but did not decrease eating disorder pathology. One might argue that this lack of an effect on the primary endpoint might be attributed to the low dosage of the present prevention program. It was the aim of the present project to develop a prevention program with a high likelihood of a long-term implementation in schools. Also, the effects observed in the present study were comparable to those observed in universal prevention programs with a higher dosage (for an overview see [14]). Thus, the efficacy of universal prevention programs for eating disorders can not only be attributed to their quantity.

Eating disorder pathology scores of male and female adolescents developed differently in the intervention and control groups as well as in grades 8 and 11. In grade 8 females, an increase in Ch-EDE scores from baseline to post-intervention and six months later was observable in both the intervention and control group (Fig. 4). No such increase in eating disorder pathology could be observed in grade 8 male adolescents from both groups. In contrast to grade 8 adolescents, the decrease in ChEDE-Q scores in 11th grade adolescents indicated that this subgroup seem to have benefited from the prevention program with regard to their eating disorder pathology. One explanation for the varying effects of the present program might be that the mechanisms of efficacy are influenced by the age of delivery. In this context, the results of the mediation analyses revealed that in grade 8 changes in eating disorder knowledge significantly mediated the relation between group allocation and changes in eating disorder psychopathology at follow-up. A positive regression coefficient indicated that 


\section{a Eating disoder pathology}

Total sample

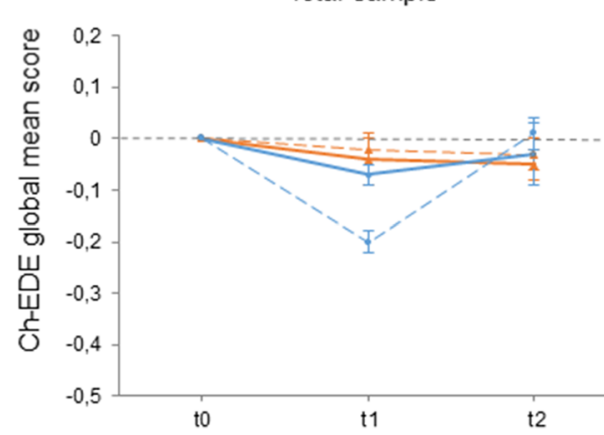

\section{Anxiety}

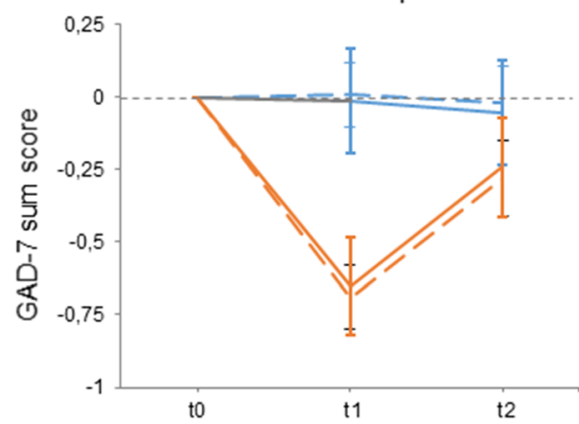

- IG, adjusted mean

\section{b Eating disorder knowledge}

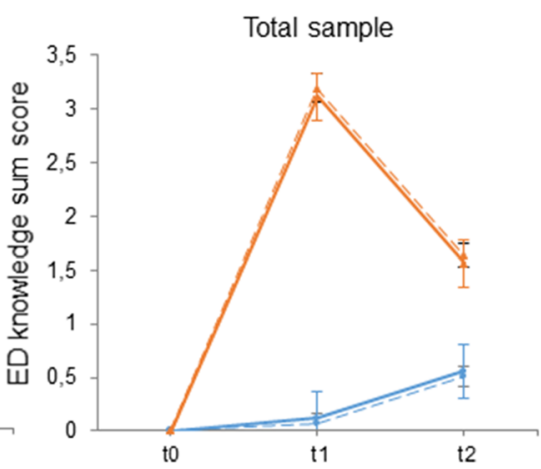

d Internalization/Pressure

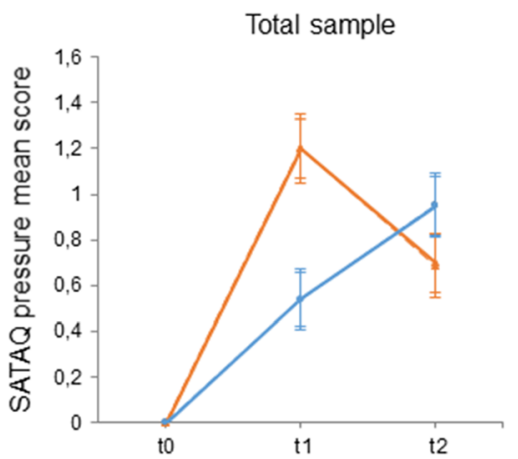

CG, adjusted mean
G, raw mean

\section{e Subgroup analyses: Eating disorder pathology}

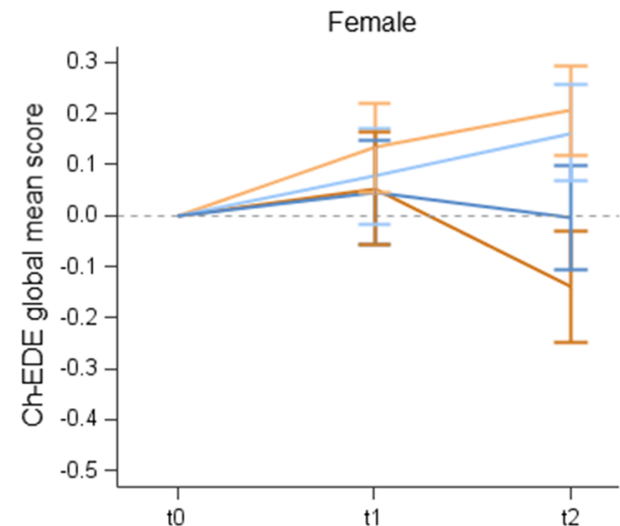

IG, 8th grade, adjusted mean
CG, 8th grade, adjusted mean

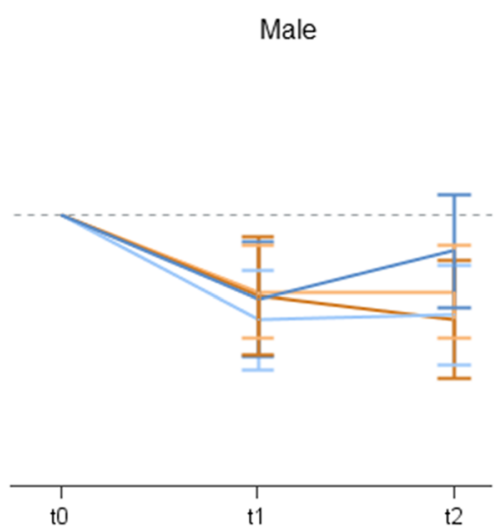

IG, 11th grade, adjusted mean
CG, 11th grade, adjusted mean

Fig. 4 Mean changes from baseline to post-intervention and six-month follow-up for the primary and selected secondary outcomes. Note. $t_{0}=$ Baseline assessment, $\mathrm{t}_{1}=$ post-intervention, $\mathrm{t}_{2}=$ six months post-intervention. a Children's Eating Disorder Examination Questionnaire global mean score (primary outcome, scale range $0-6$, negative change scores are desired); $\mathbf{b}$ Eating disorder knowledge sum score (secondary outcome, scale range 0-20, positive change scores are desired); c Generalized Anxiety Disorder Scale global sum score (secondary outcome, scale range 0-21, negative change scores are desired); d Sociocultural Attitudes Towards Appearance Questionnaire, pressure subscale sum score (secondary outcome, scale range 5-25, negative change scores are desired); a-d means are shown as raw means and adjusted for sex, grade, body mass index, baseline Ch-EDE-Q score; e Children's Eating Disorder Examination Questionnaire global mean score (post hoc analyses of primary outcome regarding significant interactions between group, grade and time as well as between sex, grade and time, scale range $0-6$, negative change scores are desired), means adjusted for sex, grade, body mass index, baseline Ch-EDE-Q score. $\mathrm{CG}=$ control group, IG = intervention group; ${ }^{*} p<0.05,{ }^{* *} p<0.01,{ }^{* * *} p<0.001$ 
a knowledge increase was associated with an increase in eating disorder psychopathology. However, a confidence interval very close to zero pointed in the direction that this effect might not be clinically relevant. A similar picture occurred with regard to perceived pressure to suit Western beauty ideals where a significantly mediating effect was observed in grade 8 but not in grade 11. In this age group no such significant mediating effects of eating disorder knowledge or perceived pressure to suit Western beauty ideals could be observed. When one compares the content of the present eating disorder prevention program with those developed by Wilksch and colleagues [16] it is noteworthy that the prevention program with the most favorable outcome (Media Smart) focused heavily on media internalization, media transported stereotypes and pressure from media whereas the programs with iatrogenic effects focused either on a healthy lifestyle (Life Smart) or included interventions addressing appearance related issues (HELLP). The present program comprised interventions comparable to all three programs. Thus, with regard to participants from grade 8 it seems reasonable for future prevention programs to conduct dismantling investigations to a) examine whether addressing media internalization is truly the most promising approach for universal prevention programs and b) whether it is favorable to focus on a small number of adressed risk factors to ensure that the content is elaborately learned rather than targeting multiple risk factors. On a meta-level our results might provide further evidence that eating disorder knowledge increase should not be an intervention goal and part of primary or secondary endpoints in universal prevention programs that address young adolescents.

The specific effect of the intervention on 11th grade participants may be regarded as significant because an increase in eating disorder pathology has to be expected particularly in late adolescent females [3] and late adolescence is the peak age of onset for bulimia nervosa and binge eating disorder [10]. Participants from grade 11 differed from grade 8 participants in that in grade 11 anxiety mediated the relation between group allocation and changes in eating disorder psychopathology at postintervention. This result is in line with previous evidence that identified anxiety as a risk factor for eating disorder symptomatology in female adolescents [43]. Furthermore, in this age group a knowledge increase did not result in an iatrogenic effect and might contribute to greater symptom awareness during later adolescence and facilitated help-seeking behavior in affected individuals and their peers [44].

Previous studies have shown that prevention programs for adolescents in the eating disorder field tend to be more effective when delivered to females or when solely addressed to either females or males who already show an elevated risk $[12,45]$. In our view, the applicability of universal prevention programs to entire classes involving both females and males through gender-adapted materials appears to be important. Such an approach can help to increase awareness of eating disorder risk factors, such as peer pressure, thereby decreasing the stigmatization of mental illnesses and improving prosocial behavior in classes for the most relevant peer group during adolescence [9]. Furthermore, a long term implementation might be facilitated if schools do not need to provide alternative activities for males.

The strengths of our study include a sound study design with cluster randomization and follow-up assessment as well as the high number of participating schools and adolescents. The high practicability of the genderadapted materials, the brevity of the intervention, and the close cooperation with an established stakeholder of school-based prevention are further strengths of this research.

The results of the study should be interpreted in light of the following limitations. First, individual randomization seemed neither feasible nor appropriate to evaluate the present program [46]. A cluster randomization of classes would have been more methodologically rigorous given that students within the same school might be more similar than compared with students from other schools. However, participating schools refused a randomization of classes. Cluster randomization with schools as the unit of randomization facilitated the implementation of the intervention and prevented control participants from being unintentionally affected by the intervention. Moreover, we considered it more suitable for students to be in their current peer group, as the present prevention materials comprised several interactive interventions, i.e., group discussions. Second, concealing the allocation of treatment from schools, students or parents was not feasible. This non-blinded allocation might have resulted in an expectancy effect. Allocation to the control group for example might have reduced schools' commitment to take part in the study, it might have influenced students' responses to the outcome measures and parents' behavior towards their children. With the aim to overcome this limitation and to ensure schools', students' and parents' commitment, the prevention program was offered to participants in the control group after the follow-up assessment. Third, we observed a low response rate of schools who were willing to participate in the present study together with a high attrition rate of schools who consented to participate. This might have decreased the representativeness of our sample in that only particularly motivated schools agreed to participate. It has to be kept in mind, however, that during the implementation of the study important changes in the Hamburgian school 
system occurred. Many schools which might have been otherwise motivated to participate in the present study were occupied with restructuring activities. Of the participating schools, only one had gone through restricting activities prior to participation. On a more general level, the low response rate of schools highlights the great need to increase the motivation of schools to implement prevention programs. Fourth, the selfreport measures were answered during classes; hence, social desirability bias may have affected the completion of these measures. We attempted to limit this potential bias through the attendance of research assistants who ensured that the questionnaires were completed discreetly and quietly.

\section{Conclusions}

In summary, the present prevention program can be recommended to older adolescents where it resulted in increased knowledge on eating disorders and a decrease in eating disorder pathology. We do believe that knowledge of mental illnesses should be an integral part of education and that prevention programs might also contribute to destigmatization and early detection of mental diseases. Future studies should focus on the evaluation of the present program in grade 11 adolescents and examine the possibility of a teacher- or peer-based delivery of the intervention.

\section{Additional files}

\section{Additional file 1: Study protocol. (PDF $402 \mathrm{~kb}$ )}

Additional file 2: Table S1. Baseline and follow-up assessment outcomes based on least-squares mean estimates of a mixed-model analysis within the intention-to-treat sample $(n=1020$ intervention participants; $n=981$ control participants). (DOCX $21 \mathrm{~kb}$ )

\section{Abbreviations}

BMI: Body-Mass-Index; ChEDE-Q: Eating Disorder Examination Questionnaire - children's version; CPA: Centre for the Prevention of Addiction; GAD7: Generalized Anxiety Disorder Scale; MSCS: Multidimensional Self-concept Scale; PHQ-9: Patient Health Questionnaire - Depression Module; SATAQ: Social Attitudes Towards Appearance Questionnaire

\section{Acknowledgements}

The study was part of a health service research project in the Hamburg Metropolitan Area (psychenet; ISRCTN44979231; subproject Anorexia and Bulimia Nervosa; Principal Investigator: Bernd Löwe]. The project psychenet aims to improve the prevention, diagnosis and treatment of people with mental illnesses.

We would like to thank all members of the Center for Prevention of Addiction from the State of Hamburg Institute for Teacher Training and School Development for their extensive cooperation. We are grateful to all teachers and students who supported the study with their participation as well as all research assistants who supported the study during recruitment. We would also like to thank Dipl.-Psych. Denise Kästner from the Department of Psychosomatic Medicine and Psychotherapy, University Medical Center Hamburg-Eppendorf \& Schön Klinik Hamburg Eilbek, for her assistance in data management and data cleaning.

\section{Funding}

The study was part of a health service research project in the Hamburg Metropolitan Area (psychenet), funded by the German Federal Ministry of Education and Research [Grant number: O1KQ1002B; Principal Investigator: Bernd Löwe]. The funders had no role in study design, data collection and analysis, decision to publish, or preparation of the manuscript.

\section{Availability of data and materials}

The datasets used and analyzed during the current study are available from the corresponding author on reasonable request.

\section{Authors' contributions}

AW and AD had full access to all data in the study, and they take responsibility for the integrity of the data and the accuracy of the data analysis. BL had the idea for the study. BL, GR, AG and KW conceived the study. AW participated in implementing the study. AW and AD performed the statistical analysis, and KW supervised the statistical analysis of the study. All authors substantially contributed to the acquisition, analysis, or interpretation of data for this work. AG and AW drafted the manuscript. All authors revised it critically for important intellectual content. All authors gave their final approval of the version to be published.

\section{Ethics approval and consent to participate}

Before providing written informed consent, students and their parents or legal guardians obtained a letter of consent with a detailed description of the allocated study condition and were, therefore, not blinded to their study condition. Eligible students were asked to provide written informed consent. Additional written informed consent from a parent or legal guardian was required for participants under 16 years of age. The study was approved by the Ethics Committee of the Psychotherapist Chamber of Hamburg, Germany (July 26, 2011), as well as the Hamburg supervisory school authority.

\section{Consent for publication}

Not applicable.

\section{Competing interests}

The authors declare that they have no competing interests.

\section{Publisher's Note}

Springer Nature remains neutral with regard to jurisdictional claims in published maps and institutional affiliations.

\section{Author details}

${ }^{1}$ Department of Psychosomatic Medicine and Psychotherapy, University Medical Center Hamburg-Eppendorf \& Schön Clinic Hamburg Eilbek, Germany, Martinistraße 52, 20246 Hamburg, Germany. ${ }^{2}$ Department of Medical Biometry and Epidemiology, University Medical Center Hamburg-Eppendorf, Germany, Martinistraße 52, 20246 Hamburg, Germany. ${ }^{3}$ Berlin University of Psychology, Am Köllnischen Park 2, 10179 Berlin, Germany. ${ }^{4}$ Department of Children and Adolescent Psychiatry, Psychosomatics and Psychotherapy, University Medical Center Münster, Germany, Schmeddingstr. 50, 48149 Münster, Germany.

Received: 2 March 2017 Accepted: 3 August 2017

Published online: 11 August 2017

\section{References}

1. Smink FR, van Hoeken D, Oldehinkel AJ, Hoek HW. Prevalence and severity of DSM-5 eating disorders in a community cohort of adolescents. Int J Eat Disord. 2014;47(6):610-9.

2. Berger U, Schilke C. Strauss B: [Weight concerns and dieting among 8 to 12 year-old children]. Psychother Psychosom Med Psychol. 2005;55(7):331-8.

3. Hölling $H$, Schlack R. Eating disorders in children and adolescents. First results of the German Health Interview and Examination Survey for Children and Adolescents (KiGGS). Bundesgesundheitsblatt Gesundheitsforschung Gesundheitsschutz. 2007:50(5-6):794-9.

4. Micali N, Ploubidis G, De Stavola B, Simonoff E, Treasure J. Frequency and patterns of eating disorder symptoms in early adolescence. J Adolesc Health. 2014;54(5):574-81. 
5. Weigel A, Rossi M, Wendt H, Neubauer K, von Rad K, Daubmann A, Romer G, Löwe B, Gumz A. Duration of untreated illness and predictors of late treatment initiation in anorexia nervosa. J Public Health. 2014;22(6):519-27.

6. Steinhausen HC. The outcome of anorexia nervosa in the 20th century. Am J Psychiatry. 2002;159(8):1284-93.

7. Rohde P, Stice E, Marti CN. Development and predictive effects of eating disorder risk factors during adolescence: Implications for prevention efforts. Int J Eat Disord. 2015;48(2):187-98.

8. Dustan CJ, Paxton SJ, McLean SA. An evaluation of a body image intervention in adolescent girls delivered in single-sex vs. co-educational classroom settings. Eat Behav. 2017;25:23-31.

9. Sharpe H, Schober I, Treasure J, Schmidt U. The role of high-quality friendships in female adolescents' eating pathology and body dissatisfaction. Eat Weight Disord. 2014;19(2):159-68.

10. Hilbert A, Pike KM, Goldschmidt AB, Wilfley DE, Fairburn CG, Dohm FA, Walsh BT, Striegel Weissman R. Risk factors across the eating disorders. Psychiatry Res. 2014;220(1-2):500-6.

11. Jacobi C, Hayward C, de Zwaan M, Kraemer HC, Agras WS. Coming to terms with risk factors for eating disorders: application of risk terminology and suggestions for a general taxonomy. Psychol Bull. 2004;130(1):19-65.

12. Stice $E$, Shaw $H$, Marti CN. A meta-analytic review of eating disorder prevention programs: encouraging findings. Annu Rev Clin Psychol. 2007;3:207-31.

13. Wilksch SM. Where did universal eating disorder prevention go? Eat Disord. 2014;22(2):184-92.

14. Bailey AP, Parker AG, Colautti LA, Hart LM, Liu P, Hetrick SE. Mapping the evidence for the prevention and treatment of eating disorders in young people. J Eat Disord. 2014;2:5.

15. Ciao AC, Loth K, Neumark-Sztainer D. Preventing eating disorder pathology: common and unique features of successful eating disorders prevention programs. Curr Psychiatry Rep. 2014;16(7):453.

16. Wilksch SM, Paxton SJ, Byrne SM, Austin SB, McLean SA, Thompson KM, Dorairaj K, Wade TD. Prevention Across the Spectrum: a randomized controlled trial of three programs to reduce risk factors for both eating disorders and obesity. Psychol Med. 2015;45(9):1811-23.

17. Wilksch SM. How can we improve dissemination of universal eating disorder risk reduction programs? Eat Behav. 2017;25:58-61.

18. Pokrajac-Bulian A, Zivcic-Becirevic I, Calugi S, Dalle Grave R. School prevention program for eating disorders in Croatia: a controlled study with six months of follow-up. Eat Weight Disord. 2006;11(4):171-8.

19. Wilksch SM, Tiggemann M, Wade TD. Impact of interactive school-based media literacy lessons for reducing internalization of media ideals in young adolescent girls and boys. Int J Eat Disord. 2006;39(5):385-93.

20. Ghaderi A, Martensson M. Schwan H: "Everybody's Different": a primary prevention program among fifth grade school children. Eat Disord. 2005; 13(3):245-59.

21. Berger U, Schaefer JM, Wick K, Brix C, Bormann B, Sowa M, Schwartze D, Strauss B. Effectiveness of reducing the risk of eating-related problems using the German school-based intervention program, "Torera", for preadolescent boys and girls. Prev Sci. 2014;15(4):557-69.

22. Russell-Mayhew S, Arthur N, Ewashen C. Targeting students, teachers and parents in a wellness-based prevention program in schools. Eat Disord. 2007;15(2):159-81.

23. McVey G, Tweed S, Blackmore E. Healthy Schools-Healthy Kids: a controlled evaluation of a comprehensive universal eating disorder prevention program. Body Image. 2007;4(2):115-36.

24. Franks A, Kelder SH, Dino GA, Horn KA, Gortmaker SL, Wiecha JL, Simoes EJ. School-based programs: lessons learned from CATCH, Planet Health, and Not-On-Tobacco. Prev Chronic Dis. 2007;4(2):A33.

25. Weigel A, Gumz A, Uhlenbusch N, Wegscheider K, Romer G, Löwe B. Preventing eating disorders with an interactive gender-adapted intervention program in schools: Study protocol of a randomized controlled trial. BMC Psychiatry. 2015;15

26. Hilbert A, Buerger A, Hartmann AS, Spenner K, Czaja J, Warschburger P. Psychometric evaluation of the eating disorder examination adapted for children. Eur Eat Disord Rev. 2013;21(4):330-9.

27. Berg KC, Peterson CB, Frazier P, Crow SJ. Psychometric evaluation of the eating disorder examination and eating disorder examination-questionnaire: a systematic review of the literature. Int J Eat Disord. 2012;45(3):428-38.

28. Knauss C, Paxton SJ, Alsaker FD. Validation of the German version of the Sociocultural Attitudes Towards Appearance Questionnaire (SATAQ-G). Body Image. 2009;6(2):113-20.
29. Argyrides M, Kkeli N, Kendeou P. Validation of the factor structure of the Greek adaptation of the Sociocultural Attitudes Towards Appearance Questionnaire (SATAQ-3). Body Image. 2014;11(3):201-5.

30. Llorente $\mathrm{E}$, Warren $\mathrm{CS}$, de Eulate LP, Gleaves DH. A Spanish version of the Sociocultural Attitudes Towards Appearance Questionnaire-3 (SATAQ-3): translation and psychometric evaluation. J Clin Psychol. 2013;69(3):240-51.

31. Rotatori AF. Multidimensional Self Concept Scale. Meas Eval Couns Dev. 1994;26(4):265-8.

32. Schütz A, Sellin I. Multidimensionale Selbstwertskala: MSWS. Göttingen: Hogrefe; 2006.

33. Kroenke K, Spitzer RL, Williams JB. The PHQ-9. J Gen Intern Med. 2001;16(9): 606-13.

34. Löwe B, Kroenke K, Herzog W, Gräfe K. Measuring depression outcome with a brief self-report instrument: sensitivity to change of the Patient Health Questionnaire (PHQ-9). J Affect Disord. 2004;81(1):61-6.

35. Löwe B, Decker O, Müller S, Brähler E, Schellberg D, Herzog W, Herzberg PY. Validation and standardization of the Generalized Anxiety Disorder Screener (GAD-7) in the general population. Med Care. 2008;46(3):266-74.

36. Spitzer RL, Kroenke K, Williams JB, Löwe B. A brief measure for assessing generalized anxiety disorder: the GAD-7. Arch Intern Med. 2006;166(10): 1092-7.

37. Levine MP. Universal prevention of eating disorders: A concept analysis. Eat Behav. 2017;25:4-8.

38. Cohen J. A power primer. Psychol Bull. 1992;112(1):155-9.

39. Senn S, Stevens L, Chaturvedi N. Repeated measures in clinical trials: simple strategies for analysis using summary measures. Stat Med. 2000;19(6):86177

40. Hayes AF. Mediation, Moderation, and Conditional Process Analysis. In: Introduction to Mediation, Moderation, and Conditional Process Analysis: A Regression-Based Approach edn. New York: Guilford Publications; 2013. p. 1-20.

41. Schafer JL, Graham JW. Missing data: our view of the state of the art. Psychol Methods. 2002;7(2):147-77.

42. Yager Z, Diedrichs PC, Ricciardelli LA, Halliwell E. What works in secondary schools? A systematic review of classroom-based body image programs. Body Image. 2013;10(3):271-81.

43. Penas-Lledo E, Bulik CM, Lichtenstein P, Larsson H, Baker JH. Risk for selfreported anorexia or bulimia nervosa based on drive for thinness and negative affect clusters/dimensions during adolescence: A three-year prospective study of the TChAD cohort. Int J Eat Disord. 2015;48(6):692-9.

44. Babitsch B, Gohl D, von Lengerke T. Re-revisiting Andersen's Behavioral Model of Health Services Use: a systematic review of studies from 19982011. Psychosoc Med. 2012;9:Doc11.

45. Watson HJ, Joyce T, French E, Willan V, Kane RT, Tanner-Smith EE, McCormack J, Dawkins H, Hoiles KJ, Egan SJ. Prevention of eating disorders: A systematic review of randomized, controlled trials. Int J Eat Disord. 2016; 49(9):833-62.

46. Campbell M, Fitzpatrick R, Haines A, Kinmonth AL, Sandercock $P$, Spiegelhalter D, Tyrer P. Framework for design and evaluation of complex interventions to improve health. BMJ. 2000;321(7262):694-6.

\section{Submit your next manuscript to BioMed Central and we will help you at every step:}

- We accept pre-submission inquiries

- Our selector tool helps you to find the most relevant journal

- We provide round the clock customer support

- Convenient online submission

- Thorough peer review

- Inclusion in PubMed and all major indexing services

- Maximum visibility for your research

Submit your manuscript at www.biomedcentral.com/submit
Biomed Central 